\title{
Avaliação da Usabilidade de Três Acionadores Computacionais
}

\author{
Usability Evaluation of Three Computational Triggers
}

\author{
Letícia Simões Ferreira ${ }^{1}$, Leslie Andrews Portes ${ }^{2}$, Vagner Rogério dos \\ Santos $^{3}$, Acary Souza Bulle Oliveira ${ }^{4}$, Francis Meire Fávero ${ }^{5}$
}

\begin{abstract}
1.Fisioterapeuta, Especialista em Neurologia Clínica e Doenças Neuromusculares, voluntária no ambulatório de Esclerose Lateral Amiotrófica do Setor de Doenças Neuromusculares, Universidade Federal de São Paulo, São Paulo-SP, Brasil.

2. Fisioterapeuta do Exercício, voluntário do Departamento de Fisiologia Cardíaca da Universidade Federal de São Paulo, São Paulo-SP, Brasil.

3.Docente Adjunto do Departamento de Oftalmologia e Ciências Visuais da Universidade Federal de São Paulo, São Paulo-SP, Brasil.

4. Neurologista, Chefe do Setor de Investigação em Doenças Neuromusculares da Universidade Federal de São Paulo, São Paulo-SP, Brasil.

5. Professora Afiliada do Departamento de Neurologia e Neurocirurgia da Universidade Federal de São Paulo, São Paulo-SP, Brasil.
\end{abstract}

\section{Resumo}

Introdução. A Usabilidade é definida como a facilidade de uso de algo, levando-se em conta a ergonomia, o esforço mental e as atitudes do usuário frente à tecnologia. Na avaliação de Usabilidade devem ser analisadas a eficiência, eficácia e satisfação do usuário na interação com a tecnologia. Uma Tecnologia Assistiva envolve os recursos que ampliam habilidades funcionais em Pessoas com Deficiência como acionadores computacionais. Objetivo. Avaliar a Usabilidade de três acionadores computacionais com a Escala de Avaliação de Tecnologia Assistiva de Comunicação Aumentativa. Método. Dez portadores de Doença do Neurônio Motor participaram de um teste de Usabilidade com os três acionadores, onde tinham que receber, instalar, programar e realizar uma tarefa proposta, e ao final, responderam a Escala. Resultados. Os três acionadores se apresentaram igualmente como "regulares" na classificação de Usabilidade segundo a Escala, sem diferença estatisticamente significante $(p=0,94)$. O principal aspecto a ser melhorado, segundo os resultados apontados na Escala, é o da embalagem dos acionadores. Conclusão. Dentre os três acionadores, não se evidenciou um que fosse melhor do que o outro em termos de Usabilidade, sendo necessários ajustes para aperfeiçoar a Usabilidade em todos.

Unitermos. Equipamentos de Autoajuda; Tecnologia de Informação; Tecnologia Educacional

\begin{abstract}
Introduction. Usability is defined as the ease of use of something, taking into account ergonomics, mental effort and user attitudes towards technology. In the Usability evaluation, the efficiency, effectiveness and user satisfaction in the interaction with the technology should be analyzed. Assistive Technology involves features that enhance functional skills in People with Disabilities as computational triggers. Objective. To evaluate the Usability of three computational triggers with the Augmentative Communication Assistive Technology Assessment Scale. Method. Ten patients with Motor Neuron Disease participated in a Usability test with the three triggers, where they had to receive, install, program and perform a proposed task, and at the end, answered the Scale. Results. The three triggers were equally "regular" in the Usability classification according to the Scale, with no statistically significant difference $(p=0.94)$. The main aspect to be improved, according to the results indicated in the Scale, is the packaging of the actuators. Conclusion.
\end{abstract}


Among the three triggers, there was no evidence that one was better than the other in terms of Usability, requiring adjustments to improve Usability in all.

Keywords. Self-Help Devices; Information Technology; Educational Technology

Trabalho realizado na Universidade Federal de São Paulo, UNIFESP, São Paulo-SP, Brasil.

Conflito de interesse: não

Recebido em: 2018

Aceito em: 2018

Endereço para correspondência: Francis Meire Fávero. Email: ffave.nexp@latoneuro.com.br

\section{INTRODUÇÃO}

A Usabilidade é definida como uma qualidade que está relacionada à facilidade de uso de algo ${ }^{1}$ proporcionando melhorias na qualidade de interação do usuário com determinada interface, sendo que à medida que a tecnologia avança, exige-se que ela apresente maior eficiência e organização².

A Usabilidade deve ser baseada no desempenho do usuário com ênfase na facilidade de uso e no grau de aceitação do produto $^{3}$, sendo que para o usuário, um bom produto é aquele que atende às suas necessidades, é fácil de usar, não induz de soluções equivocadas mas que possua atividades organizadas e uma metodologia específica ${ }^{4}$.

Quando o termo usabilidade está relacionado ao produto, as características ergonômicas deste devem ser enfatizadas; já quando relacionada ao usuário, devem ser levados em conta o esforço mental e as atitudes do usuário frente ao produto $^{3}$, sendo que para o usuário, um bom produto é aquele que atende às suas necessidades, é fácil de usar, não induz de soluções equivocadas mas que 
possua atividades organizadas e uma metodologia específica 4 .

Normalmente os avaliadores de Usabilidade são profissionais de áreas diversas, possuem alguma experiência em estatística, com interesse em Usabilidade através de experiências em gerenciamento de produtos, escrita técnica, suporte ao cliente ou outros papéis relacionados ${ }^{5}$.

As métricas relacionadas aos testes de avaliação de Usabilidade envolvem a eficiência, eficácia e satisfação, sendo a eficiência relacionada ao conceito de produtividade, em função do trabalho realizado por unidade de tempo, como o tempo gasto na tarefa; a eficácia referente ao quão bem o usuário é capaz de executar a tarefa, em termos de até que ponto o objetivo é alcançado e a satisfação sendo determinada a partir de medidas subjetivas que são administradas na conclusão da tarefa ou no final do teste de usabilidade 5 .

Tecnologia Assistiva (TA) é um termo utilizado para denominar o arsenal de recursos e serviços que proporcionam ou ampliam habilidades funcionais em Pessoas com Deficiência $(P C D)^{6}$, tendo como objetivos principais promover maior independência, Qualidade de Vida (QV), inclusão social, permitindo ao indivíduo ampliar sua comunicação, facilitar mobilidade, controlar o ambiente e suas habilidades de aprendizado e trabalho ${ }^{7}$.

Dentre as categorias de uma TA, encontram-se os Recursos de Acessibilidade do Computador, que incluem 
softwares e hardwares que tornam o computador acessível a pessoas com limitações como de fala? .

Os acionadores podem ser simples - retangulares ou circulares - e que conectados a mouse plus atuam como o clique do mouse, ou acionam brinquedos ou softwares.

O acionador PENSO é funciona por meio da pressão exercida em uma bolsa de ar que captura o movimento e transforma em sinal sonoro. Simulando o apertar de uma tecla, ele interage com o computador sendo configurável ao usuário, tanto ergonomicamente quanto ao nível de estímulo físico ${ }^{8}$.

Este acionador é dividido em três partes: 1 . Bolsa de ar: onde os pacientes exercerão a pressão para acionamento; 2 . Sensor de pressão: componente eletrônico que mensura a pressão exercida em atuação ou em repouso; 3. Dispositivo eletrônico: entende as informações do sensor de pressão e toma as atitudes desejadas ${ }^{8}$.

$O$ acionador adaptativo por piscadela A-blinX detecta $O$ piscar dos olhos, por meio de um sensor posicionado na armação de qualquer óculos, comum ou com lentes, emite um sinal sonoro a cada piscada detectada, permitindo comandar interfaces para computador via usb ${ }^{9}$.

O Teclado Inteligente Multifuncional (TIX) consiste em um equipamento assistivo que substitui o teclado e o mouse através de um painel eletrônico com onze teclas sensíveis ao toque ${ }^{10}$. Com a combinação de duas teclas é possível obter todas as funcionalidades de um teclado e mouse convencional. 
A EAUTACA, inicialmente denominada como Protocolo de Avaliação de Usabilidade, foi desenvolvida no idioma Português no Brasil em 2016 e aperfeiçoada em 2018, com o objetivo de avaliar as dificuldades e facilidades do usuário de tecnologia assistiva ao receber, instalar, programar e usar o dispositivo.

A escala EAUTACA possui um manual de preenchimento, com informações sobre cada item de cada um dos cinco domínios, bem como o significado dos valores a serem pontuados como sendo de 0 a 3 baseados na escala Likert ${ }^{11}$. Este manual auxiliará no preenchimento da escala EAUTACA.

Cada domínio possui determinado número de itens. Cada item deve ser respondido de acordo com a percepção do usuário frente à tecnologia. A pontuação vai de 0 a 3 sendo que valor igual ou próximo a zero representa negatividade, ausência de algo ou muita dificuldade, e valor igual ou próximo a 3 representa positividade, presença de algo ou facilidade. Para preencher, basta assinalar com um " $x$ " na linha e coluna correspondente em cada item. No final da escala, contém a classificação do escore total, permitindo identificar o grau de Usabilidade da tecnologia.

Com isso, esta pesquisa tem como objetivo avaliar a usabilidade de três acionadores computacionais mediante a Escala de Avaliação de Usabilidade de Tecnologia Assistiva de Comunicação Aumentativa (EAUTACA), criada pelos pesquisadores, identificando possíveis diferenças 
significativas entre eles e apontar qual tecnologia apresenta melhor Usabilidade.

\section{MÉTODO}

\section{Amostra}

Este estudo é do tipo observacional com segmento transversal e foi desenvolvido no Setor de Investigações de Doenças Neuromusculares (SIDNM) da disciplina de Neurologia Clínica do Departamento de Neurologia/Neurocirurgia da Universidade Federal de São Paulo (UNIFESP). Este estudo foi submetido e aprovado pelo Comitê de Ética em Pesquisa, CAAE no 91992718.3.0000.5505 da UNIFESP/Hospital São Paulo.

Foram selecionados aleatoriamente dez pacientes com Doença do Neurônio Motor, sendo cinco mulheres e cinco homens, com média de idade de 54,4 anos, sendo que um caso apresentava quadro clínico somente de acometimento bulbar, seis casos apresentavam comprometimento bulbar e apendicular e três, apenas comprometimento apendicular.

Os critérios de inclusão selecionavam os pacientes com pontuação acima de 21 pontos no Mini Exame do Estado Mental (MEEM) com diagnóstico de Doença do Neurônio Motor, e com movimentos preservados de membros superiores ou piscar de olhos. Foram excluídos os pacientes com comprometimento cognitivo que impedisse a compreensão das orientações e utilização de equipamentos de tecnologia computacional. O MEEM foi desenvolvido nos 
Estados Unidos da América (EUA) e publicado em 1975, com o objetivo de avaliar o estado mental e sintomas de demência ${ }^{12}$.

\section{Procedimento}

Foram utilizados três modelos de acionadores computacionais: Acionador PENSO! da Olivas ${ }^{\circledR}$ + bexigas; Acionador a-blinX da GERAES ${ }^{\circledR}$, + óculos sem lente; Teclado Inteligente Multifuncional TIX da GERAES $®$.

Em um período de dez meses foram realizadas triagens com os pacientes que aguardavam atendimento médico no SIDNM. A triagem era baseada na pontuação do MEEM onde apenas os pacientes que seguiam os critérios de inclusão seguiam para as demais etapas da pesquisa. Após assinarem o Termo de Consentimento Livre e Esclarecido (TCLE), os pacientes eram levados até 0 laboratório de pesquisa, onde eram apresentados os dois acionadores que seriam avaliados por eles.

Caso o paciente não possuísse nenhum conhecimento em uso de computadores, o pesquisador Ihe ensinava quanto ao uso do teclado virtual do Windows associado a página de digitação do Word. Foram informados sobre a função dos acionadores e que eles deveriam com a ajuda de seus cuidadores, instalar e utilizá-lo associado ao teclado virtual para digitar a palavra "AMOR" estipulada como tarefa do teste. 
Caso apresentassem alguma dificuldade na instalação e utilização dos acionadores, o pesquisador interviria com auxílio para ser possível dar sequência no teste.

Após se familiarizar com o processo de digitação com o modo varredura no teclado do Windows, a gravação era iniciada e o paciente juntamente com o cuidador iniciavam a instalação e utilização. Foi estabelecido que o paciente testasse um acionador de cada vez e ao final de cada avaliação, preenchesse a Escala referente ao acionador testado.

\section{RESULTADOS}

Após a aplicação dos testes, coleta e análise dos dados, foi possível verificar que as três tecnologias se apresentam igualmente como regulares na classificação de Usabilidade segundo a escala EAUTACA (Figura 1). Segundo os resultados, não houve diferença entre as tecnologias $(p=0,94)$.

Conforme os resultados obtidos dos domínios A, B, e $E$, as três tecnologias se classificaram igualmente como Ruim, Boa e Excelente respectivamente. Nos demais domínios, $C$ e $D$, apesar de as tecnologias apresentarem classificações diferentes, não houve diferença, sendo o domínio $C(p=0,73)$ e o domínio $D(p=29)$. 
Figura 1. Escores de Usabilidade por Domínios.
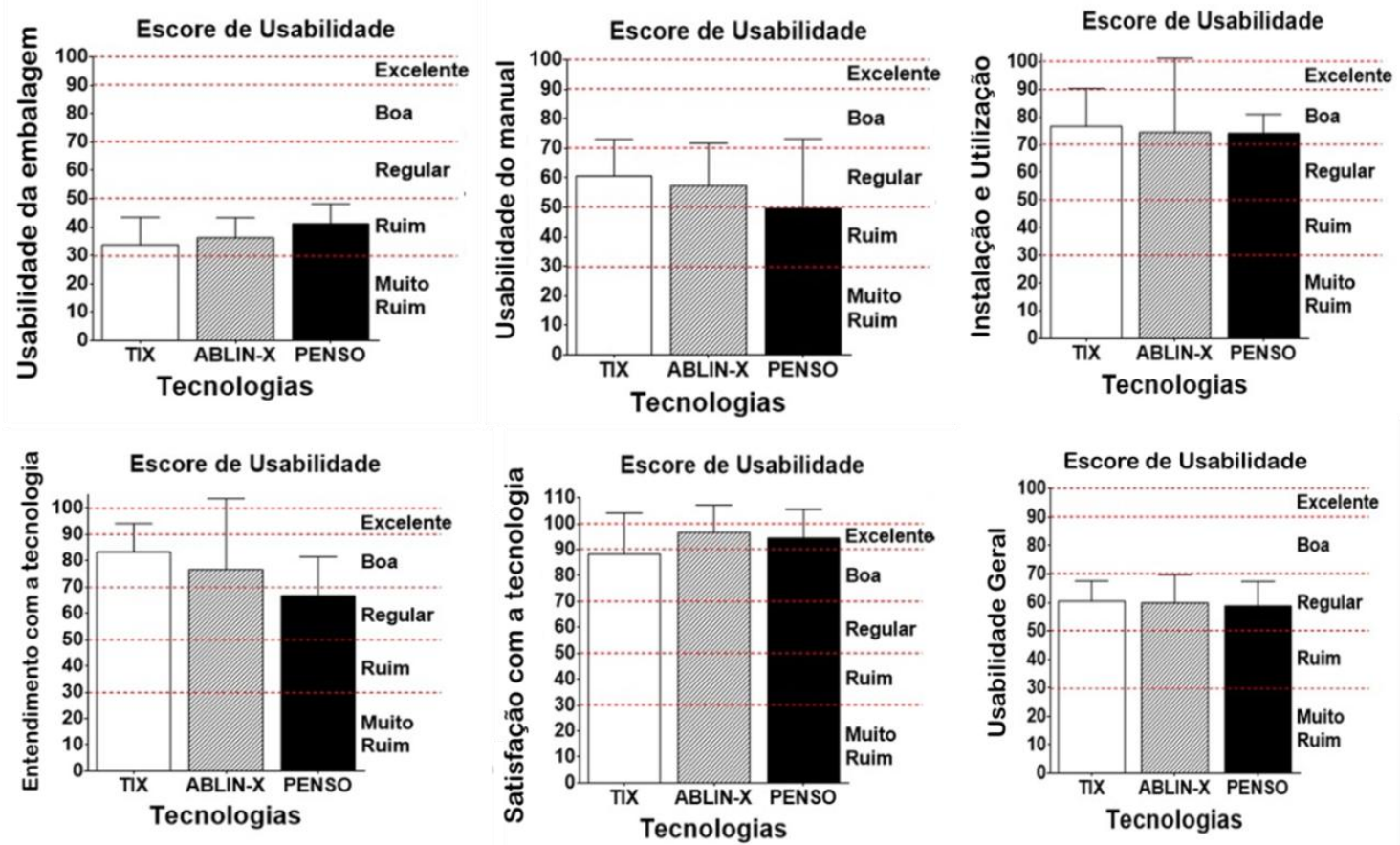

Escores de Usabilidade (0-100\%) das três tecnologias (TIX, ABLIN-X, PENSO). Resultados apresentados por Domínios e Geral das tecnologias respectivamente $(p=0,94)$.

\section{DISCUSSÃO}

Diante do crescente avanço tecnológico é importante que os dispositivos tenham acoplados ao seu sistema operacional, ou disponibilizem em suas lojas virtuais aplicativos adicionais que auxiliam as pessoas com deficiência no manuseio e na realização de tarefas ${ }^{13}$.

Em conformidade com esta pesquisa, encontrou-se na literatura que para o processo de avaliação da usabilidade, devem ser utilizados métodos dirigidos a especialistas e a usuários, com avaliações de desempenho quantitativas e qualitativas $^{14}$. Não há nada que substitua a possibilidade de ver o usuário tentando utilizar o produto ${ }^{15}$. 
Os critérios para se avaliar a Usabilidade mediante suas métricas são: conclusão da tarefa e qualidade do resultado - para a Eficácia; desvios do caminho, quantidade ou taxa de erros, tempo para a realização da tarefa e carga mental - para Eficiência ${ }^{15}$; e análise qualitativa com entrevistas e questionários, ou análise quantitativa por meio de escala como de Likert $^{11}$ - para Satisfação.

Outra ressalva, diz respeito a realizar o teste no ambiente natural do usuário, onde as posturas assumidas por ele serão mais reais do que em laboratório ${ }^{16}$. No entanto, isto demanda maior do tempo e dispêndio financeiro para os pesquisadores se deslocarem até os usuários, também não podendo controlar fatores externos que possam inviabilizar o teste ${ }^{14}$.

O Código de Defesa do Consumidor determina como direitos do usuário a informação adequada e clara sobre os produtos e serviços, especificando a quantidade e preço, bem como sobre os riscos que apresentem ${ }^{17}$. Esses fatores foram evidenciados nas respostas do domínio $A$, onde estes aspectos da embalagem das três tecnologias foram considerados "ruins" pelos usuários.

Com relação ao domínio $\mathrm{B}$, referente ao manual de instruções, a NR12.125 ao $12.129^{18}$ regulamenta 5 itens do conteúdo mínimo que um Manual de Instruções deve conter: 
- Os equipamentos devem conter o manual de instruções fornecido pelo fabricante, com informações relativas à segurança em todas as fases de utilização;

- Serem escritos na língua portuguesa, com caracteres de tipo e tamanho que possibilitem a melhor legibilidade possível, acompanhado das ilustrações explicativas;

- Ter linguagem objetiva, clara, sem ambiguidades;

- Ter sinais ou avisos destacados referentes à segurança;

- Permanecer disponíveis a todos os usuários nos locais de trabalho.

Com base nestes itens, as questões do domínio B abrangem os principais pontos de um manual adequado e diante das respostas foi possível analisar concordantemente que de forma geral, o manual de instruções das três tecnologias foi considerado "bom".

Vale ressaltar que a maioria dos pacientes desta pesquisa estavam acompanhados de familiares ou cuidadores no momento do teste, o que influenciou diretamente nas respostas de certas questões com relação ao manuseio e instalação da tecnologia, pois estes foram realizados pelas mãos dos acompanhantes. Isso pode justificar a alta pontuação qualitativa dada pelos pacientes da fase de instalação.

No domínio $\mathrm{E}$, onde as questões eram relacionadas à satisfação do usuário, as três tecnologias foram consideradas excelentes pelos pacientes. A implantação de melhorias nos processos proporciona um aumento na 
qualidade do produto e, consequentemente, influenciam na satisfação do usuário ${ }^{19}$.

\section{CONCLUSÃO}

A níveis de Usabilidade geral, os acionadores PENSO, ABLIN-X e TIX foram considerados regulares, mediante 0 modelo de classificação proposto na escala EAUTACA desenvolvida. O processo de instalação e entendimento da tecnologia ABLIN-X se mostrou melhor, em comparação as demais, porém esses resultados não apresentaram diferença significante. Portanto, dentre os três acionadores, não se evidenciou um que fosse melhor do que o outro em termos de Usabilidade.

\section{AGRADECIMENTOS}

Programa de Apoio a Pós-Graduação e à Pesquisa Científica e Tecnológica em Tecnologia Assistiva no Brasil (PGPTA) - Edital No 59/2014.

\section{REFERÊNCIAS}

1.Nielsen J, Loranger $\mathrm{H}$. Usabilidade na web, projetando websites com qualidade. Rio de Janeiro: Elsevier, 2006. 432p.

2.Costa EM, Marques ÉV. Usabilidade: um estudo da percepção de qualidade no comércio eletrônico brasileiro. XXXV Encontro da ANPAD, 2011, p17.

3.Dias C. Usabilidade na web: criando portais mais acessíveis. Rio de Janeiro: AltaBooks, 2007, 296p.

4.Cybis W, Holtz A, Faust R. Ergonomia e usabilidade. Conhecimentos, métodos e aplicações. $3^{a}$. edição, São Paulo: Novatec, 2017, 48p.

5.Goldberg JH, Wichansky AM. Eye Tracking in usability evaluation. A practitioner's guide. Rio de Janeiro: Elsevier, 2003;493-516.

6.Bersch R, Tonolli JC. Introdução ao conceito de tecnologia assistiva e modelos de abordagem da deficiência. Porto Alegre: Centro 
Especializado em Desenvolvimento Infantil, 2006.

7.Bersch R. Tecnologia assistiva. Porto Alegre: CORDE, 2013, p140.

8.Ramos AR. "Penso": facilitar a comunicação digital a deficientes físicos (endereço na internet). Portugal: Jornal de Referência (acessado em: 2017). Disponivel em: https://jornalreferencia.wordpress.com/2017/06/29/penso-facilitar-acomunicacao-digital-a-deficientes-fisicos/

9.Assistiva GT. O que o a-blinX faz? (endereço na Internet). Belo Horiaonte: TIX (acessado em: 2018). Disponivel em: http://tix.geraestec.com.br/a-blinx/

10.GERAES. Manual de instruções do TIX (endereço na internet). Belo Horizonte: TIX (acessado em: 2018). Dsiponível em: http://tix.geraestec.com.br/.

11.Likert R. A technique for the measurement of attitudes. Arch Psychol 1932;22:1-55.

12.Folstein MF, Folstein SE, McHugh PR. Mini-mental state: a practical method for grading the cognitive state of patients for the clinician. J Psychiatr Res 1975;12:189-98. http://dx.doi.org/10.1016/00223956(75)90026-6

13.Borges VF, Mendes EG. Usabilidade de aplicativos de tecnologia assistiva por pessoas com baixa visão. Rev Bras Ed Esp 2018;24:483-500.

65382418000500002

14.Cabral AKPS, Sanguinetti DCM, Amaral DS, Marcelino JFQ, Martins LB, Costa JAP. Usabilidade de produtos de tecnologia assistiva para atividades de vida diária de pessoas com Doença de Parkinson. Ergodesign HCI 2017;5:104-15.

http://dx.doi.org/10.22570/ergodesignhci.v5iEspecial.363

15.Jordan PW. An Introduction to Usability. CRC Press (Taylos \& Francis Group), 1998, 136p.

16.Domiciano CLC, Henriques F, Ferrari DV, Crenitte PAP. Design para Pessoas: o caráter social do design gráfico por meio de experiências em pesquisa e projetos. In: Andrade ABP, Medola FO, Paschoarelli LC, Landim PC, Domiciano CLC, Henriques F, et al. Ensaios em Design. Ações inovadoras. Bauru: Editora Canal 6, 2016, p.238-65.

17.Presidência da República. Casa Civil. LEI No 8.078, DE 11 DE SETEMBRO DE 1990. Código de Defesa do Consumidor (endereço na internet). Disponível em:

http://www.planalto.gov.br/ccivil 03/leis/l8078.htm

18.Área SST - Saúde e Segurança do Trabalho. NR12 - Segurança no trabalho em máquinas e equipamentos (endereço na internet). Brasil: Ministério do Trabalho (publicada em 1978. acessada em 2015). Disponível em: https://areasst.com/nr-12-seguranca-no-trabalhoem-maquinas-e-equipamentos/

19.Ahren D, Clouse A, Turner R. A Practical Introduction to Integrated Process Improvement. Boston: Addison-Wesley Pro, 2008, 288p. 